\title{
Proteina C-reativa ultra-sensivel em pacientes com diagnóstico de doença arterial coronariana estabelecido por angiografia
}

\author{
High-sensitivity C-reactive protein in patients with angiographically defined coronary artery disease
}

Luciana Moreira Lima'; Maria das Graças Carvalho²; Andréia Assis Loures-Vale; Cirilo Pereira da Fonseca Neto4; José Carlos de Faria Garcia ${ }^{4}$; Jamil Abdala Saad ${ }^{4}$; Marinez Oliveira Sousa ${ }^{2}$

\section{unitermos \\ Angiografia coronariana}

Doença arterial coronariana

Proteina C reativa ultra-sensivel

\section{resumo}

A proteína C-reativa (PCR) é uma proteína de fase aguda, sintetizada pelo fígado em resposta às citocinas, que reflete inflamação ativa sistêmica. A inflamação tem um papel potencial no início, progressão e desestabilização das placas de ateroma. Marcadores plasmáticos de inflamação crônica têm sido consistentemente associados ao risco de doença arterial coronariana (DAC), sendo a proteína C-reativa ultra-sensível (PCRus) o marcador mais estudado. O presente estudo teve como objetivo determinar os níveis plasmáticos da PCRus de um grupo de indivíduos submetidos à angiografia coronariana, buscando estabelecer a possível correlação entre esse parâmetro e a gravidade da DAC. Níveis plasmáticos da PCR foram determinados em amostras de sangue de 17 indivíduos com ausência de ateromatose nas coronárias (controles), 12 pacientes apresentando ateromatose leve/moderada e 28 com ateromatose grave, utilizando-se o conjunto diagnóstico Biotécnica Proteína C Reativa Turbidimetria com metodologia ultra-sensível específica para monitoramento em cardiologia, com linearidade de 0,1 a $15 \mathrm{mg} / \mathrm{l}$. Não foram encontradas diferenças estatisticamente significativas entre as médias dos três grupos para o parâmetro avaliado, porém as médias obtidas para os grupos ateromatose leve/moderada e ateromatose grave permaneceram acima da faixa de referência indicada pelo método para monitoramento em cardiologia $(0,1$ a $2,5 \mathrm{mg} / \mathrm{dl})$. As médias obtidas nos três grupos apresentaram elevação crescente dos níveis plasmáticos de PCRus a partir do grupo controle, aumentando com a gravidade da aterosclerose coronariana, o que poderia sugerir a progressão do estado inflamatório em função da lesão aterosclerótica.
C-reactive protein (CPR) is an acute phase protein, synthesized by the liver in response to cytokines, and reflects active inflammation. Inflammation has a potential role in atherosclerosis triggering and progression. Plasma markers of chronic inflammation have been consistently associated to the risk of coronary artery disease (CAD), being high-sensitivity C-reactive protein the marker most studied. The aim of the present study was to determine the high-sensitivity C-reactive protein plasma levels in a group of subjects undergoing coronary angiography, trying to establish a possible correlation between this parameter and the severity of the CAD. High-sensitivity C-reactive protein plasma levels had been determined in blood of 17 subjects with no atheromatosis (controls), 12 subjects presenting mild/moderate atheromatosis and 28 subjects presenting severe atheromatosis, using Biotechnical Reactive C-Protein Turbidimetric Kit with specific high-sensitivity methodology for Cardiology, with linearity to 0.1 up $15 \mathrm{mg} / \mathrm{l}$. Significant differences between the means of the three groups were not observed, however the mean values of mild/moderate atheromatosis and severe atheromatosis had remained above the reference values used in Cardiology (0.1-2.5mg/dl). The mean values of the three groups presented an increasing rise from the control group to the severe atherosclerosis, suggesting inflammatory progression due to atherosclerotic injury. key words

Coronary angiography

Coronary artery disease

High-sensitivity C-reactive

protein

1. Doutorando da Faculdade de Farmácia da Universidade Federal de Minas Cerais (UFMG).

2. Doutora do Departamento de Análises Clínicas e Toxicológicas da Faculdade de Farmácia da UFMG.

3. Médica cardiologista do Departamento de Aterosclerose do Hospital SOCOR.

4. Médico cardiologista do Departamento de Hemodinâmica do Hospital SOCOR.

Trabalho realizado no Departamento de Análises Clínicas e Toxicológicas da Faculdade de Farmácia da UFMG e apresentado sob a forma de pôster no 32ㅜㅡongresso Brasileiro de Análises Clínicas, Coiânia, 2005. 


\section{Introdução}

A doença arterial coronariana (DAC) apresenta origem multifatorial, considerando que nenhum fator de risco a ela relacionado, se analisado de forma isolada, é estritamente essencial ou suficiente para seu desencadeamento. Quanto maior o número ou a gravidade dos fatores de risco observados, maior a probabilidade de morbidade e mortalidade precoces. A relação entre os fatores de risco parece ser de multiplicação, e não simplesmente de $\operatorname{adição}^{(11)}$. O reconhecimento dos fatores implicados no desenvolvimento de DAC se faz essencial, uma vez que permite melhor compreensão de sua patogênese e direciona a elaboração de planos preventivos e terapêuticos.

As diretrizes do Programa Nacional de Educação sobre o Colesterol dos Estados Unidos ${ }^{(2)}$ reconheceram diferentes marcadores de risco coronariano. Eles foram classificados como relacionados aos hábitos de vida (obesidade, sedentarismo e dieta aterogênica) e aos fatores de risco emergentes, incluindo os de natureza lipoprotéica, como a lipoproteína (a) $[(\mathrm{Lp}(\mathrm{a})]$ e a lipoproteína de baixa densidade (LDL) oxidada; os fatores metabólicos, como a resistência à insulina e os níveis de homocisteína; os fatores hematológicos, como os níveis do fator VII, ativador do plasminogênio tipo tecidual (t-PA) e inibidor do ativador do plasminogênio tipo 1 (PAl-1) e os marcadores de inflamação, como a proteína C-reativa ultra-sensível (PCRus).

A PCR é uma proteína de fase aguda, sintetizada pelo fígado em resposta às citocinas, que reflete inflamação ativa sistêmica. A inflamação tem papel potencial no início, na progressão e na desestabilização das placas de ateroma. Assim que os macrófagos se infiltram na parede vascular, elaboram citocinas que modulam a migração, a proliferação e a função de células inflamatórias ${ }^{(8)}$. Marcadores plasmáticos de inflamação crônica têm sido associados ao risco de DAC, sendo a PCR o marcador mais estudado ${ }^{(12)}$. O desenvolvimento de dosagens de PCRus tem sido instrumento na exploração do papel desse marcador na predição de um possível evento vascular, pois sua habilidade para prever futuros eventos coronarianos em homens e mulheres aparentemente saudáveis tem sido demonstrada por pesquisas recentes ${ }^{(3,6,7)}$.

Koenig et al. (1999)(2) demonstraram que modestas elevações nas concentrações plasmáticas de PCRus podem prever futuros eventos coronarianos. Essas observações fortalecem a associação entre inflamações de baixo grau e progressão e complicações da aterosclerose. Dados epidemiológicos prospectivos demonstraram que a atividade inflamatória sistêmica associa-se à incidência de eventos cardiovasculares em populações de indivíduos saudáveis e portadores de doença aterosclerótica ${ }^{(5)}$.

A PCRus tem sido consistentemente associada a risco cardiovascular, e sua determinação parece ser de utilidade na estratificação do risco de eventos coronarianos, porém essa determinação para estimativa do risco cardiovascular não se aplica a indivíduos fumantes, obesos, diabéticos, ou em uso de estrógenos ou antiinflamatórios, ou à presença de infecções ${ }^{(10)}$. O presente estudo teve como objetivo determinar os níveis plasmáticos da PCRus de um grupo de indivíduos submetidos à angiografia coronariana, buscando estabelecer a possível correlação entre esse parâmetro e a gravidade da DAC.

\section{Material e métodos}

Foram selecionados, no departamento de Hemodinâmica do Hospital SOCOR, em Belo Horizonte, 57 indivíduos na faixa etária de 40 a 65 anos: 17 com ausência de ateromatose nas coronárias (controles), 12 apresentando ateromatose leve/moderada e 28 com ateromatose grave. As amostras de sangue venoso foram obtidas com o paciente em jejum de 12 horas, utilizando-se tubos de vácuo sem anticoagulante. As amostras foram centrifugadas a $2.500 \mathrm{rpm}$ por $10 \mathrm{~min}$ para obtenção do soro.

A determinação quantitativa da PCRus foi realizada no soro através do uso do conjunto diagnóstico Biotécnica ${ }^{\circledR}$ Proteína C-Reativa Turbidimetria, cujo princípio analítico é o método turbidimétrico com látex aprimorado, seguindo as instruções fornecidas pelo fabricante, com metodologia ultra-sensível específica para monitoramento em cardiologia, com linearidade de 0,1 a $15 \mathrm{mg} / \mathrm{l}$. A presença da PCR na amostra causa aglutinação das partículas de látex cobertas com anticorpos anti-PCR. O grau de aglutinação é proporcional à concentração de PCR na amostra e pode ser medido por turbidimetria. Esse processo baseia-se na detecção ótica de partículas muito pequenas suspensas em meio líquido. Quando o anticorpo anti-PCR e a amostra são misturados, formam-se imunocomplexos. A diluição adquire turbidez, que é proporcional à quantidade de antígeno.

O ensaio foi realizado utilizando-se o aparelho Cobas Mira Plus ${ }^{\circledR}$ em sistema automatizado, utilizando o procedimento técnico recomendado pelo fabricante, aumentando o volume de amostra e o padrão para $50 \mu$ e usando o calibrador diluído 1:10 com água deionizada. Foi utilizado o soro controle Serodos ${ }^{\circledR}$ (In Vitro ${ }^{\circledR}$ Diagnóstica) para verificar o desempenho do ensaio.

\section{Resultados}

A análise estatística foi feita utilizando-se o teste de análise de variância (ANOVA). Os programas Sigma Stat ${ }^{\circledR}$ versão 1.0 e Prism ${ }^{\circledR}$ versão 3.0 foram utilizados para realizar as análises e elaborar o gráfico, respectivamente. Valor de $p$ menor que 0,05 foi considerado estatisticamente significativo. Não foram encontradas diferenças estatisticamente significativas entre as médias dos três grupos para o parâmetro avaliado, porém as médias obtidas para os grupos ateromatose leve/moderada e ateromatose grave se mostraram acima da faixa de referência indicada pelo método 
para monitoramento em cardiologia $(0,1$ a $2,5 \mathrm{mg} / \mathrm{dl})$. A Figura ilustra a distribuição dos valores encontrados para os três grupos estudados.

\section{Discussão}

Recentes pesquisas, estudando os componentes celulares e moleculares envolvidos no desenvolvimento e na progressão da aterosclerose, têm demonstrado que se trata de um processo dinâmico e inflamatório(8). A PCRus, considerada um marcador de inflamação sistêmica, tem sido consistentemente associada ao risco cardiovascular, e sua determinação parece ser útil na estratificação do risco de eventos coronarianos ${ }^{(2,4,9)}$. Reações inflamatórias na placa aterosclerótica presente nas coronárias contribuem para a sua instabilidade e ruptura, representando um papel importante na patogênese de eventos aterotrombóticos agudos fatais ou não ${ }^{(2)}$.

Neste estudo, os dados obtidos com as determinações plasmáticas da PCRus não apresentaram diferenças estatisticamente significativas entre os três grupos avaliados, porém suas médias permaneceram acima da faixa de referência indicada pelo método $(0,1$ a $2,5 \mathrm{mg} / \mathrm{l})$ para monitoramento em cardiologia. A análise da Figura permite observar que as médias obtidas nos três grupos apresentaram elevação crescente dos níveis plasmáticos de PCRus a partir do grupo controle $(4,3 \pm 2,9)$, aumentando com a gravidade da aterosclerose coronariana $(10,7 \pm 12,5$ para o grupo ateromatose leve/moderada e $12,8 \pm 15,5$ para o grupo ateromatose grave), o que poderia sugerir a progressão do estado inflamatório em função da lesão aterosclerótica. Entretanto, o fato de que os grupos não apresentaram diferenças significativas entre si pode ser explicado pelos altos valores de desvio padrão encontrados nos grupos ateromatose leve/ moderada e ateromatose grave (Figura). A presença de dois valores extremamente elevados, um no grupo ateromatose leve/moderada (45 mg/l) e outro no ateromatose grave $(64,9$ $\mathrm{mg} / \mathrm{l}$ (Figura), exerceu influência significativa nas médias e desvios padrão desses grupos. Valores extremos influenciam muito a média, mas pouco a mediana, que é o valor que divide a distribuição ao meio, avaliando a aglomeração dos dados do grupo. A análise das medianas obtidas para os três grupos (3,6 para o grupo controle; 4,9 para o grupo ateromatose leve/moderada e 5,65 para o grupo ateromatose grave) permite também inferir sobre um aumento crescente nos níveis de PCRus de acordo com a progressão da aterosclerose coronariana, porém sem diferenças estatisticamente significativas entre os grupos estudados.

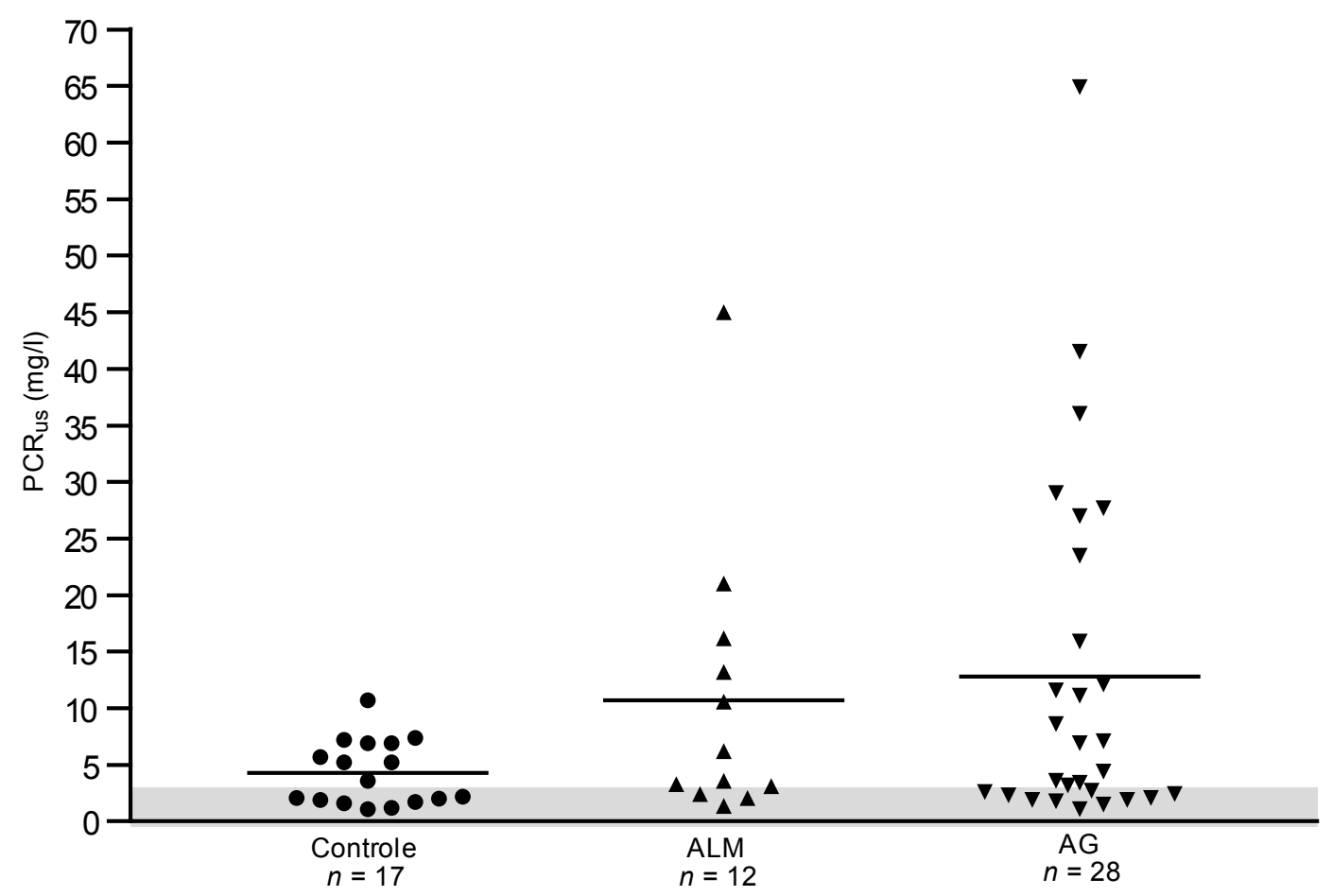

Figura - Distribuição dos valores de PCRus

Valores expressos em mg/l para os grupos controle, ALM e AG. A área sombreada corresponde à faixa de normalidade (0,1 a 2,5mg/l), e as linhas horizontais representam as médias dos grupos (4,3 2,9 para o grupo controle, 10,7 $\pm 12,5$ para o grupo ALM e 12,8 $\pm 15,5$ para o grupo AG).

PCRus: proteína C-reativa ultra-sensivel; ALM: ateromatose leve/moderada; AG: ateromatose grave. 
Tanto a média quanto a mediana obtidas para PCRus no grupo controle encontram-se superiores ao limite máximo do valor de referência (Figura). O Consenso Brasileiro sobre Prevenção da Aterosclerose ${ }^{(10)}$ considera de alto risco indivíduos com valores de PCRus acima de $1,9 \mathrm{mg} / \mathrm{l}$. A alta prevalência de hipertensão arterial $(82,4 \%)$ no grupo controle pode ter contribuído para o fato de este estudo ter demonstrado níveis elevados de PCRus nesse grupo. Segundo o mesmo consenso, a determinação da PCRus para estimativa do risco cardiovascular não se aplica a fumantes, obesos, diabéticos, entre outros fatores, não se referindo à hipertensão arterial. Saito et al. $(2003)^{(9)}$ relataram níveis significativamente mais altos de PCRus em pacientes em tratamento para hipertensão, como é o caso de 14 indivíduos do grupo controle $(n=17)$. A prevalência de hipertensão arterial nos grupos ateromatose leve/moderada e ateromatose grave também foi alta, $75 \%$ e $85,7 \%$, respectivamente. Porém, tanto as médias quanto as medianas encontradas para os níveis plasmáticos de PCRus nesses dois grupos apresentaram-se ainda mais elevadas que o limite máximo de referência indicado pelo método e maiores que a média e a mediana obtidas para o grupo controle, sugerindo que há uma tendência ao aumento progressivo nos níveis plasmáticos de $\mathrm{PCRus}$ à medida que a aterosclerose coronariana evolui, independente da presença de hipertensão arterial, que foi altamente prevalente nos três grupos, porém não apresentando diferença significativa entre eles.

\section{Conclusão}

As médias obtidas nos três grupos apresentaram elevação crescente dos níveis plasmáticos de PCRus a partir do grupo controle, aumentando com a gravidade da aterosclerose coronariana, o que poderia sugerir a progressão do estado inflamatório em função da lesão aterosclerótica.

\section{Agradecimentos}

À Fundação de Amparo à Pesquisa de Minas Gerais (FAPEMIG), à Fundação Coordenação de Aperfeiçoamento de Pessoal de Nível Superior (CAPES), ao Laboratel Laboratório de Análises Clínicas e à Biotécnica Ind. Com. Ltda. pelo apoio recebido.

\section{Referências}

I. AMERICAN HEART ASSOCIATION. Third Report of the National Cholesterol Education Program (NCEP): Expert Panel on Detection, Evaluation, and Treatment of High Blood Cholesterol in Adults (Adult Treatment Panel III). JAMA, v. 285, p. 2486-97, 2001.

2. KOENIG, W. et al. C-reactive protein, a sensitive marker of inflammation, predicts future risk of coronary heart disease in initially health middle-aged men. Circulation, v. 99, p. 237-42, 1999.

3. LIBBY, P. et al. Inflammation and atherosclerosis. Circulation, v. I05, p. I |35-43, 2002.

4. LUC, G. et al. C-reactive protein, interleukin-6 and fibrinogen as predictors of coronary heart disease. Arterioscler Thromb Vasc Biol, v. 23, p. |255-6।, 2003.

5. PEARSON,T.A. et al. Markers of inflammation and cardiovascular disease. Application to clinical and public health practice: a statement for healthcare professionals from the Centers for Disease Control and Prevention and American Heart Association. Circulation, v. 107, p. 499-51।, 2003.

6. RIDKER, P. M. et al. Comparison of C-reactive protein and low- density lipoprotein cholesterol levels in the prediction of first cardiovascular events. N Engl J Med, v. 347, n. 20, p. I557-65, 2002.

7. RIFAI, N.; RIDKER, P.M. High-sensitivity C-reactive protein: a novel and promising marker of coronary heart disease. Clin Chem, v. 47, p. 403-1।, 200 I.

8. RUSSEL, R. Mechanisms of disease: atherosclerosis an inflammatory disease. N Eng J Med, v. 340, p. 15-126, 1999.

9. SAITO, M. et al. Relations of plasma high-sensitivity C-reactive protein to traditional cardiovascular risk factors. Atherosclerosis, v. 167, p. 73-9, 2003.

10. SOCIEDADE BRASILEIRA DE CARDIOLOGIA. III Diretrizes Brasileiras Sobre Dislipidemias e Diretrizes de Prevenção da Aterosclerose. Arq Bras Cardiol, v. 77, supl. III, p. I-48, 200 I.

I I.WORLD HEALTH ORGANIZATION. Health topics. Disponível em: <http://www.who.int/cardiovascular_diseases/en/>. Acesso em: 6 nov. 2004.

12. YU, H.; RIFAI, N. High-sensitivity C-reactive protein and atherosclerosis: from theory to therapy. Clin Biochem, v. 33, p. $601-10,2000$. 